\title{
Editorial
}

\section{Epidemiología de la malaria en Costa Rica}

\section{Generalidades}

La malaria es una enfermedad causada por un parásito del género Plasmodium. Es trasmitida por la picadura de mosquitos del género Anopheles. Existen más de 150 especies de Plasmodium que infectan diferentes vertebrados, pero solo cuatro ( $P$. falciparum, $P$. vivax, $P$. ovale y $P$. malariae) infectan al hombre. Las dos especies más comunes son: el $P$. falciparum, que tiene una distribución global, pero es más común en África y es la especie más agresiva; causa la muerte principalmente por coma o por anemia. El $P$. vivax, también de distribución mundial, puede causar infecciones debilitantes y recurrentes, pero rara vez la muerte.

Únicamente las hembras se alimentan de sangre (son hematófagas), por lo que son las responsables de la transmisión de la enfermedad; tienen hábitos nocturnos o crepusculares. Son capaces de infectarse y de permitir el ciclo esporogónico completo de Plasmodium. Cuando el mosquito pica a una persona infectada, los parásitos se multiplican sexualmente (esporogonia) en el tubo digestivo y se desarrollan en las glándulas salivares; el mosquito al inocular los parásitos en un nuevo huésped; estos colonizan primero el hígado, donde tienen varios ciclos de multiplicación asexuada y de donde salen a invadir los eritrocitos. Dentro de los eritrocitos, los parásitos se reproducen en forma asexuada (esquizogonia), y esta multiplicación es la responsable de los síntomas. Algunos parásitos, dentro de los glóbulos rojos, se transforman en gametocitos, que son las formas sexuadas de Plasmodium. Cuando el mosquito Anopheles ingiere la sangre infectada, los gametocitos se diferencian en su intestino y reinician, por reproducción sexuada, el ciclo biológico.

\section{La malaria en el mundo}

La malaria no es un problema exclusivo de los países tropicales, sino que es global y afecta más de 100 países (Figura 1). Los cambios de clima (con el subsiguiente aumento de la temperatura ambiente) y los movimientos poblacionales pueden alterar el mapa de distribución.

Más de 2.500 millones de personas viven en riesgo de adquirir la enfermedad. Entre $300 \mathrm{y}$ 500 millones de personas se enferman de malaria anualmente y mueren cerca de 3 millones por año. Cada minuto, de 3 a 5 niños mueren de malaria. Cada hora, la malaria ocasiona más decesos que los que produjo la epidemia de EBOLA en Zaire en 1995. Sin embargo, la malaria no es reconocida en el primer mundo como una catástrofe de salud pública, como sí ha ocurrido con el SIDA o el EBOLA, a pesar de que tiene una tasa de muerte por año superior a la de muertes por SIDA durante los últimos 15 años.

\section{La malaria en Costa Rica}

Costa Rica ha sido un país de baja incidencia malárica, la mayoría de los casos se concentran en la Región Huetar Atlántica, específicamente en el cantón de Matina. Como puede notarse en la figura 2, la incidencia de la malaria empezó a reducirse en el país a partir de 1999, pero en 2005 se observó un repunte que podría ser peligroso si no se controla.

En el contexto del proyecto "Programa Regional de Acción y Demostración de Alternativas Sostenibles para el Control de Vectores de Malaria sin el uso del DDT en México y Centroamérica", promovido por la OPS/OMS, en el Área Demostrativa del Cantón de Talamanca y el Área Rectora de Salud de Matina se está desarrollando, con apoyo técnico del IRET-UNA, un sistema

ISSN 0001-6002/2008/50/2/72-74 Acta Médica Costarricense, (C2008 Colegio de Médicos y Cirujanos de información geográfica con datos epidemiológicos y ambientales relativos al control de vectores de la malaria. En 2006, el Ministerio de Salud empezó a desarrollar nuevas acciones concretas que se dividieron en tres aspectos específicos: 


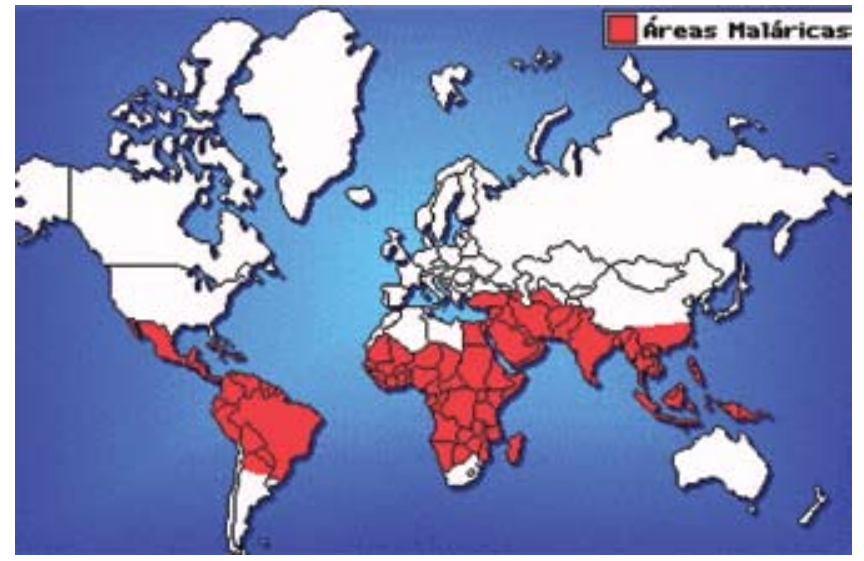

Figura 1. Áreas maláricas.

1. Control del vector: Por medio de la alianza con sectores internos y externos al sector Salud y el concurso de la empresa pública y privada, se realizaron estrategias de fumigación dentro y fuera de las viviendas; destrucción de criaderos, abatización de los suampos y drenaje en sitios de aguas estancadas. Se logró identificar las casas maláricas y sus características: hendijas en las paredes, poco aseo de la vivienda y personal (el mosquito es atraído por el sudor corporal); sin uso de productos de fumigación residual dentro de las viviendas y ausencia de mosquiteros.

2. Diagnóstico microbiológico: Se actualizó la capacitación de los funcionarios en las lecturas de las laminillas y se designó una cantidad mayor para el reporte oportuno de los exámenes.

3. Seguimiento de los casos: Se reforzó la visita domiciliaria de aquellas personas positivas, asegurándose de que el tratamiento fuera tomado de acuerdo con la normativa vigente; se dio seguimiento a la negativización por medio de la gota gruesa y se mejoró el diagnóstico de los casos nuevos de manera oportuna.

Las medidas anteriores empezaron a surtir efecto y ya en ese mismo año se notó una reducción significativa de casos, tendencia que se mantuvo en 2007. Figura 3.

\section{Situación 2007}

En 2007 se reportaron 1223 casos de malaria, lo que significó una disminución de 1680 casos (-57.9\% variación relativa) respecto a los 2903 reportados en 2006.

De las 22.641 muestras examinadas, 1223 (5.4\%) resultaron positivas; de estas 1212 (99.1\%) correspondieron a $P$. vivax, 10 mixtas $(0.82 \%)$ a $P$. vivax y solo una de $P$. falciparum.

La Región Huetar Atlántica, con 1145 casos, representa el $93.6 \%$ del total del país; notándose un decremento de

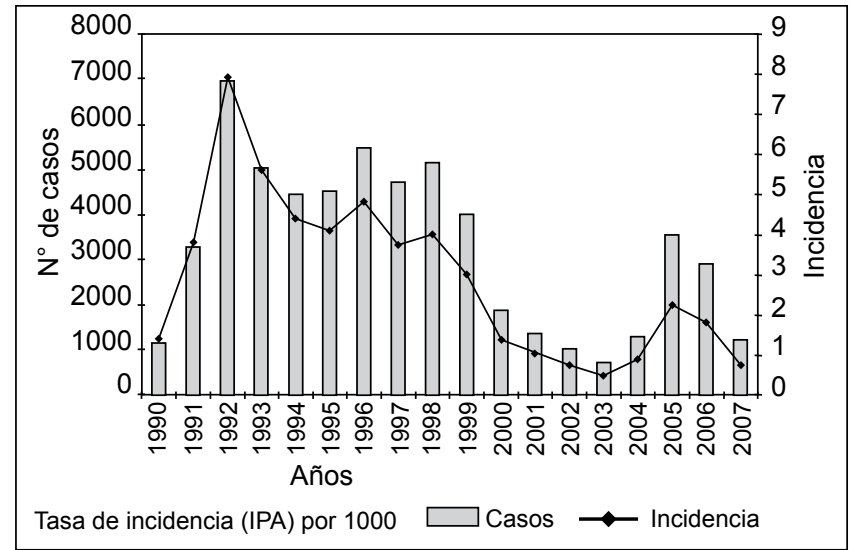

Figura 2. Casos de malaria y tasas de incidencia anual. Costa Rica. Periodo 1990-2007.

1662 casos respecto a 2006 ( $-59.2 \%$ variación relativa). El cantón de Matina tuvo un decremento de 1.518 casos respecto a 2006 ( $-64.1 \%$ variación relativa), con 850 casos reportados, que representan el $74.2 \%$ de la región y el $70.0 \%$ del país. El cantón de Limón, con 142 casos, y el de Pococí, con 77 , representan el $12.4 \%$ y el $6.7 \%$, respectivamente, de los casos de la región. En estos tres cantones se concentra el $87.4 \%$ de la malaria del país.

En la Región Huetar Norte, zona fronteriza de gran vulnerabilidad y receptividad malarigénica, solo se diagnosticaron 23 casos, lo que representa el 1.9\% de la malaria del país, con una disminución de 19 casos con respecto a los reportados en 2006 (- 45.2\% variación relativa). Los cantones de San Carlos, con 17, y Los Chiles, con 6 casos, aportan el 100\% de la incidencia de la malaria en esa región.

La contribución de casos del resto de las regiones se desglosa de la siguiente manera: Chorotega $4(0.3 \%)$, Pacífico Central 7 (0.6\%), Central Norte 7 (0.6\%), Brunca $26(2.1 \%)$ y fuera del área malárica 11 ( 0.9\%).

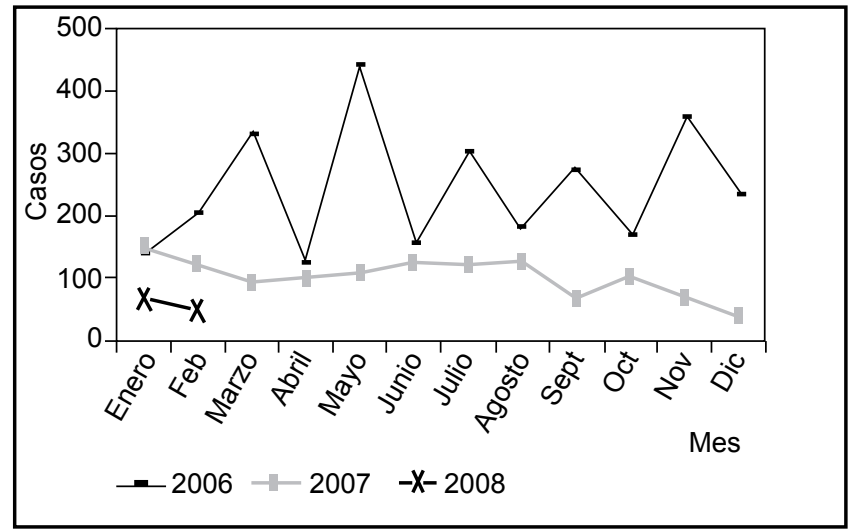

Figura 3. Casos de malaria por mes. Costa Rica 2006-2007-2008. 


\section{Situación 2008}

A la semana epidemiológica 09 (finalizada el 01 de marzo), se han registrado 115 casos de malaria; el 97,4\% pertenece a la Región Huetar Atlántica; al comparar los casos de malaria registrados por región en 2008 con los de 2007 a la misma semana epidemiológica, se observa un decremento de casos en el país del 58,3\%. Como puede notarse en la figura 4 , la reducción de casos se ha dado de manera sostenida, lo que augura éxitos para la estrategia de intervención.

\section{Conclusiones:}

La malaria, como enfermedad y como proceso natural, se debe analizar tomando en consideración las influencias del entorno, el parásito, el mosquito y la evolución del proceso salud - enfermedad. De especial importancia son las acciones de promoción, prevención, tratamiento $\mathrm{y}$ rehabilitación, con el fin de influir sobre la realidad social como agentes de cambio para mejorar la calidad de vida del ciudadano y la sociedad.

Históricamente, la palabra malaria proviene del "mal aire" de los sitios cenagosos donde se reproduce el mosquito vector. La malaria ha afectado al hombre desde hace milenios, y hoy es una de las enfermedades infecciosas de mayor prevalencia en el mundo. El control debe ubicarse en diversos contextos, como son los factores que influyen en la transmisión y propagación de la enfermedad, pues este ente que se conforma por vector, parásito, huésped, ambiente y condiciones determinantes (economía, política y sociedad) se manifiesta en la aparición de la enfermedad y en algunos casos en el surgimiento de epidemias.

Los puntos críticos de control de la infección son: proveer un pronto acceso al tratamiento efectivo; prevenir y controlar la malaria durante el embarazo; controlar los vectores sin la utilización de insecticidas que alteren el ambiente; tratar con efectividad las situaciones de emergencia y epidemia, y ver la enfermedad como un problema social que requiere la acción conjunta de políticas estatales, actitudes e iniciativas sociales y eficacia, además de la preparación de las entidades medicas.

El avance en el control de la malaria en nuestro país ha sido exitoso, pero debe ser sostenible, para ello se requiere dotar a las áreas rectoras y de salud de los insumos necesarios para llevar adelante los diversos programas. Además, se precisa educación médica continua, educación a la comunidad global y personal y, sobre todo, un sólido conocimiento de la epidemiología nacional.

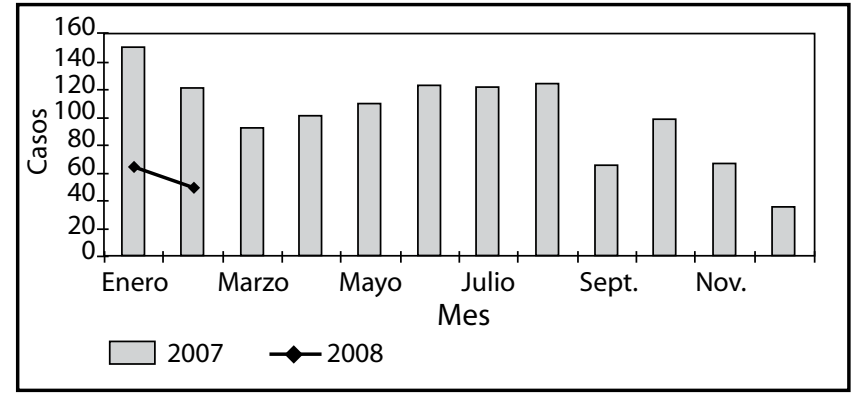

Figura 3. Casos de malaria por mes. Costa Rica 2007-2008.

Reconocimientos: Al Dr. Willy Carrillo y la Dra. Teresita Solano, de la Dirección de Vigilancia de la Salud; al Dr. Roger Brown, la Dra. Yorleny Molina y el Dr. Rodrigo Martín, de la Dirección Regional y Área Rectora de la Región Huetar Atlántica; a la Dra. Nidia Calvo y la Dra. Liliana Jiménez, del INCIENSA, al equipo de la CCSS y a todos los funcionarios de Control de Vectores, por el impresionante trabajo lleno de mística, entusiasmo, creatividad y conocimientos técnicos, que ha permitido llevar con éxito el plan de control de malaria.

María Luisa Ávila-Agüero

Ministra de Salud

\section{Referencias:}

1. Nahlen BL, Low-Beer D. Building to collective impact: the Global Fund support for measuring reduction in the burden of malaria. Am J Trop Med Hyg. 2007;77 (S6):321-7.

2. Nantulya FN, Kengeya-Kayondo JF, Ogundahunsi OA. Research themes and advances in malaria research capacity made by the Multilateral Initiative on Malaria. Am J Trop Med Hyg. 2007;77(S61):303-13.

3. Alilio MS, Bygbjerg IC, Breman JG. Are multilateral malaria research and control programs the most successful? Lessons from the past 100 years in Africa. Am J Trop Med Hyg. 2004;71(S2):268-78.

4. Cibulskis RE, Bell D, Christophel EM, Hii J, Delacollette C, Bakyaita $\mathrm{N}$, Aregawi MW. Estimating trends in the burden of malaria at country level. Am J Trop Med Hyg. 2007;77(S61):133-7.

5. Wongsrichanalai C, Barcus MJ, Muth S, Sutamihardja A, Wernsdorfer WH. A review of malaria diagnostic tools: microscopy and rapid diagnostic test (RDT). Am J Trop Med Hyg. 2007;77(S6):119-27.

6. White NJ. How antimalarial drug resistance affects post-treatment prophylaxis. Malar J. 2008;11;7:9.

7. Gosling RD, Drakeley CJ, Chandramohan D. Effective malaria control: better burden estimates needed. Lancet. 2008;371:724.

8. Maher B. Malaria: the end of the beginning. Nature. 2008;451:1042-6.

9. Hopkin M. Malaria: the big push. Nature. 2008;451:1047-9.

10. Tanner M, de Savigny D. Malaria eradication back on the table. Bull World Health Organ. 2008;86:82. 